\title{
Methylthioadenosine promotes remyelination by inducing oligodendrocyte differentiation
}

\author{
Beatriz Moreno 1,2, Gemma Vila1', Begoña Fernandez-Diez', Raquel Vázquez', Alessandra di Penta ${ }^{1,3}$, Oihana Errea',
} Nagore Escala ${ }^{1}$, Andrés Miguez ${ }^{4}$, Jordi Alberch ${ }^{4}$ and Pablo Villoslada ${ }^{1,5,6^{*}}$ (D)

\begin{abstract}
Background: Methylthioadenosine is a metabolite of the polyamine pathway that modulates methyltransferase activity, thereby influencing DNA and protein methylation. Since methylthioadenosine produces neuroprotection in models of inflammation, ischemia and epilepsy, we set out to evaluate the role of methylthioadenosine in promoting remyelination, a process that will protect axons in demyelinating diseases and that will aid functional recovery.

Methods: The effect of methylthioadenosine in promoting remyelination was tested in mouse cerebellum organotypic cultures that were exposed to lipopolysaccharide to induce neuroinflammation, or lysolecithin to induce chemical demyelination. In addition methylthioadenosine administration was also tested in vivo, using the cuprizone model of demyelination. The molecular pathways involved in this methylthioadenosine activity were evaluated in primary cortical mouse astrocytes.

Results: In models of neuroinflammation or chemical demyelination, methylthioadenosine prevented the loss of myelin and promoted remyelination in vitro by increasing the number of mature myelinating oligodendrocytes.

Methylthioadenosine enhanced myelin production in the cuprizone model, in conjunction with a clinical improvement. Methylthioadenosine enhanced STAT-3 phosphorylation in astrocytes in vitro, and the production of ciliary neurotrophic factor (CNTF), a trophic factor known to promote oligodendrocyte maturation and differentiation, as well as remyelination.

Conclusions: The remyelination promoted by methylthioadenosine suggests a role for the polyamine pathway in oligodendrocyte maturation and survival, paving the way for new therapeutic strategies to promote regeneration in Multiple Sclerosis and other demyelinating diseases.
\end{abstract}

Keywords: Methylthioadenosine, Remyelination, Oligodendrocyte, Neuroprotection, Multiple sclerosis, Demyelinating diseases, Therapy

\section{Background}

Multiple Sclerosis (MS) is an autoimmune disease characterized by Central Nervous System (CNS) demyelination, oligodendrocyte damage and axonal loss [1]. Current treatments have focused on preventing inflammation and they have limited efficacy on the progressive phase. Remyelination is considered to be a promising way to protect neurons and restore functionality, a therapeutic strategy for MS that

\footnotetext{
*Correspondence: pvilloslada@clinic.ub.es; Pablo.VillosladaDiaz@ucsf.edu ${ }^{1}$ Center of Neuroimmunology, Institut d'Investigacions Biomediques August Pi Sunyer (IDIBAPS), Barcelona, Spain

${ }^{5}$ University of California, San Francisco, USA

Full list of author information is available at the end of the article
}

is being actively pursued $[2,3]$. The myelin-axon unit is critical to maintain the trophism of long axons, and for the exchange of metabolites and macromolecules like lactate, $\mathrm{N}$ acetyl Aspartate or lipids [4]. This metabolic relationship further reinforces the attractiveness of remyelination as a therapeutic strategy to preventing axon loss in the later stages of the disease [5]. Yet even if remyelination would have been efficient in the early stages of MS its effects might be limited in the long term, particularly given the resistance of adult oligodendrocyte precursor cells (OPCs) to differentiate into myelinating oligodendrocytes (OLs) [6] and the molecular changes in demyelinated axons that lead to the rejection of myelin (e.g. PSA-NCAM re-expression) [7]. 
Methylthioadenosine is a natural metabolite of the polyamine pathway and it serves many functions in cell metabolism: driving the regeneration of adenosine and methionine stocks; modulating gene expression (e.g. activating serine-threonine phosphatase 1 that leads to the dephosphorylation of SR proteins), proliferation, differentiation and apoptosis; or inhibiting protein and DNA methylation by competing with $\mathrm{S}$-adenosylmethionine for methyltransferases $[8,9]$. Methylthioadenosine is a selective inhibitor of protein arginine methyltransferase 5 (PRMT5) activity $[10,11]$, which in turn dampens cRAF methylation and degradation; this cRAF being responsible for increasing MEK1-2 and STAT3 phosphorylation [12]. Methylthioadenosine also exhibits immunomodulatory activity by suppressing the production of pro-inflammatory cytokines and enhancing the production of antiinflammatory cytokines through an interaction with the nuclear factor kappa B (NFkB) pathway. Interestingly, such activity produces benefits in models of autoimmune diseases like MS $[13,14]$. Finally, methylthioadenosine displays neuroprotective activity, preventing neuronal damage in models of ischemia, epilepsy and Parkinson's disease [15].

In light of these protective effects of methylthioadenosine, we set out to evaluate the role of methylthioadenosine in promoting OPC maturation and OL myelination in vitro and in vivo using different models of demyelination. We found that this metabolite increases the number of mature myelinating OLs. Thus, methylthioadenosine may prove to be beneficial as a therapeutic agent to manage demyelinating diseases.

\section{Methods}

\section{In vivo studies}

\section{Animals}

All animals handling was carried out in accordance with the European Council Directive (2010/63/EU) and the Spanish regulations for the procurement and care of experimental animals (1201 RD/2005, October 10). The animal protocols used were approved by the Ethical Committee on Animal Research at the University of Barcelona.

\section{Treatments}

Methylthioadenosine (Sigma) was dissolved in phosphate buffered saline (PBS) at a concentration of $12 \mathrm{mg} / \mathrm{ml}$ and mice received a daily intraperitoneal (i.p.) injection at a dose of $60 \mathrm{mg} / \mathrm{kg}$. Placebo animals were injected daily with PBS alone i.p.

\section{Cuprizone demyelinating mouse model}

We fed 2-month-old C57BL/6J mice of either sex with $0.2 \%$ (wt/wt) cuprizone (Sigma Aldrich) for 4, 5 or 6 weeks (Additional file 1: Figure S1). The animals were then allowed to recover for 7,8 or 9 weeks before methylthioadenosine $(60 \mathrm{mg} / \mathrm{kg})$ or the placebo were injected daily (ip) as preventive or curative treatments. Methylthioadenosine dose was selected from previous animal studies in experimental autoimmune encephalomyelitis [14].

\section{Sensory motor behavior testing}

Animals were trained in the rotarod using constant speed (18rpm) 3x per day during 8 days with a rest of 5 min between each trial. Animals were tested during the remyelination phase after cuprizone treatment twice a week.

\section{In vitro studies}

\section{Primary cortical mouse astrocytes}

Cortical astrocytes were obtained from P3 C57BL6 wildtype mouse pups (of either sex) after dissection of the cortex and removal of the meninges. The tissue obtained was dissociated, plated in $75 \mathrm{~cm}^{2}$ flasks in NM-15 conditioned medium (MEM 1X, Gibco; 15\% fetal bovine serum -FBS-, Gibco; $90 \mathrm{mM} \mathrm{D-glucose,} \mathrm{Sigma)} \mathrm{and}$ maintained in an incubator at $37^{\circ} \mathrm{C}$ in an atmosphere of $5 \% \mathrm{CO}_{2}$. After 5 days in vitro (DIV), the cells were purified by agitating in an orbital shaker for $16-18 \mathrm{~h}$ at 250 rpm, discarding the detached cells and incubating the adherent cells for a further two DIV in fresh medium. Astroglial cells were replated into 6-well plates and cultured to confluence. Prior to performing the experiments, NM-15 medium was replaced with DMEM/F12 (Gibco) serum free medium and the cells were left for 48 h. The cells were then treated with Lipopolysaccharide (LPS) $(10 \mu \mathrm{g} / \mathrm{ml}, \mathrm{L4391}$; Sigma) overnight and methylthioadenosine $(192 \mu \mathrm{M})$ or placebo was added the following day for the ELISA and PCR studies (24 h), or it was maintained over the next 5 days for xMAP phosphorylation assays (samples collected 15, $30 \mathrm{~min}$ or $1 \mathrm{~h}$ after the last treatment was added).

\section{Cerebellar organotypic mouse cultures}

Cerebellar organotypic cultures were established as described previously [16]. Parasagittal slices $(300 \mu \mathrm{m})$ of the postnatal day 7 (P7) C57BL/6 mouse cerebellum (of either sex) were obtained with a tissue slicer (McIIwain), the slices were placed in medium on collagen-coated Millicell-CM culture semiporous inserts $(0.4 \mu \mathrm{m}$; Millipore, Bedford, MA, USA) and they were kept for up to 3 weeks in an atmosphere of $5 \% \mathrm{CO}_{2}$ at $37^{\circ} \mathrm{C}$ in $50 \%$ basal medium containing: Earle's salts (GIBCO), 25\% Hank's balanced salt solution (GIBCO), 25\% heat inactivated horse serum, $5 \mathrm{mg} / \mathrm{ml}$ glucose, $0.25 \mathrm{mM}$ L-glutamine, and $25 \mu \mathrm{g} / \mathrm{ml}$ penicillin/streptomycin. To induce neuroinflammation, LPS (15 $\mu \mathrm{g} / \mathrm{ml}, \mathrm{L} 4391$; Sigma) was added to the organotypic cultures 7 days after preparing the inserts. Methylthioadenosine or a placebo were added $24 \mathrm{~h}$ 
before LPS and the samples were collected $24 \mathrm{~h}$ after LPS addition for Myelin Basic Protein (MBP) and Neurofilament (NFL) staining, and $0,1,3,6,12,24,48,72$ and $96 \mathrm{~h}$ after the addition of LPS for IL-1 $\beta$, TNF- $\alpha$, iNOS and CNPase detection. For chemical demyelination, lysolecithin $(0.5 \mathrm{mg} / \mathrm{ml}, \mathrm{L} 4129$; Sigma) was added 7 days after insert preparation and the cultures were incubated overnight $\left(14\right.$ h.) at $37^{\circ} \mathrm{C}$. Subsequently, the medium containing lysolecithin was replaced with fresh medium and methylthioadenosine or saline were added the next day and every 2 days thereafter until the tissue was analyzed after 9, 13, 17 and 21 DIV. All control cultures analyzed were time matched with the treated cultures and LLL12 (partial and reversible inhibitor of STAT3 phosphorylation, Ref: 1792-5 Deltaclon) was used at $2.5 \mu \mathrm{M}$ and $5 \mu \mathrm{M}$.

\section{Western blotting}

Expression of inducible nitric oxide synthase (iNOS) and 2',3'-cyclic-nucleotide 3'-phosphodiesterase (CNPase) was assessed in Western blots of mouse cerebellum organotypic slices pre-treated with methylthioadenosine (48 $\mu \mathrm{M}$ or $192 \mu \mathrm{M})$ or the placebo, and stimulated with LPS $(15 \mu \mathrm{g} / \mathrm{ml})$ at different time points $(0,1,3,6,12,24$, 48, 72 and $96 \mathrm{~h}$ ), as described previously [16]. The Western blots were probed with a mouse antibody against CNPase (Abcam) and a rabbit antibody against iNOS (BD Bioscience).

\section{Enzyme-linked immunosorbent assay (ELISA)}

Cerebellar organotypic cultures were pre-treated with methylthioadenosine $(48 \mu \mathrm{M}$ or $192 \mu \mathrm{M})$ or a placebo at seven DIV and they were then stimulated with LPS $(15 \mu \mathrm{g} / \mathrm{ml})$ at different time points $(0,1,3,6,12,24,48$, 72 and $96 \mathrm{~h}$ ). The culture supernatants were collected to quantify interleukin $1 \beta$ (IL-1 $\beta$ ), tumor necrosis factor (TNF- $\alpha$ ) and CNTF using mouse enzyme-linked immunosorbent assay (ELISA) kits according to the manufacturer's instructions (eBioscience, San Diego, CA, USA).

\section{Immunostaining}

Immunostaining was performed as previously published [16]. After fixation with 4\% paraformaldehyde, cerebellar slices were removed gently from the inserts and they were blocked for $2-4 \mathrm{~h}$ at room temperature in PBS + $10 \%$ goat serum $+1 \%$ bovine serum albumin $(\mathrm{BSA})+$ $0.2 \%$ Triton X-100. The sections were incubated overnight at $4^{\circ} \mathrm{C}$ with the primary and secondary antibodies diluted in PBS $+1 \%$ goat serum $+1 \%$ BSA $+0.2 \%$ Triton $\mathrm{X}-100$. After each overnight incubation, the slices were washed at room temperature three times in PBS $+0.05 \%$ Triton X-100, each wash lasting $1 \mathrm{~h}$. Finally, the slices were mounted on slides, coverslipped and analyzed on a confocal microscope (LSM 510; Zeiss). Primary antibodies against the following proteins were used for immunostaining: myelin basic protein (MBP, Rat antiMBP 82-87 antibody; Serotec), Neurofilament light (NFL, anti-NF-L C28E10; Cell Signaling), glial fibrillary acidic protein (GFAP, rabbit anti-GFAP; DakoCytomation), oligodendrocyte transcription factor 2 (Olig 2, rabbit antiOlig 2; Santa Cruz Biotechnology) and CNTF (rabbit polyclonal anti-CNTF; Abcam). The secondary antibodies used were: Cy2-linked mouse immunoglobulin G (IgG), Cy3-linked rabbit IgG (from goat, 1:200; GE Healthcare, Freiburg, Germany) and Fluor 488 Goat anti-Rat IgG Alexa (1:200; Molecular Probes, Eugene, OR, USA).

\section{Image analysis}

A confocal microscope (Laser Scanning Confocal Microscope Leica SP2) was used to obtain stacks of photographs of MBP and NFH immunolabelling at $1 \mu \mathrm{m}$ intervals in the organotypic cultures $\times 40$ magnification. Slices thinned after culture to approximately $30 \mu \mathrm{m}$ thickness (depending on the number of days in culture). Myelinated fibers are best observed between a depth of 5 to $20 \mu \mathrm{m}$ from the upper surface and this is the reason why our results were taken from this level. Myelinated neurofilaments were quantified as the percentage of axons stained with NfL with MBP surrounding sheaths respect to the total number of axons (without MBP sheaths).

Percentage of the area immunoreactive for Olig2 or MBP antibodies was measured in $\times 40$ magnification images acquired by confocal microscopy. The range of the slice was determined using Z-stack imaging at 1- $\mu \mathrm{m}$ intervals and a series of images derived from Z-stack imaging were analyzed. Briefly, binary masks were defined using the same cut-off intensity threshold value for each region of interest, which corresponds to each cell immunostained, defined as the minimum intensity because of specific staining above background values. Then, the percentage of the area occupied by Olig2 or MBP was measured automatically using ImageJ software in each cerebellum organotypic tissue. Results are given by averaging values determined in at least four separate microscopic fields from six different slices. MBP immunoreactivity of the brain stained sections was quantified after obtaining the integrated densities of the midline corpus callosum from the different animals ( $n=6$ per group).

\section{Electron microscopy}

Cerebellar slices were fixed for $24 \mathrm{~h}$ in $2 \%$ paraformaldehyde and $2.5 \%$ glutaraldehyde in $0.1 \mathrm{M}$ PBS at $4{ }^{\circ} \mathrm{C}$, and they were then washed in $0.1 \mathrm{M}$ PBS for $12 \mathrm{~h}$ and postfixed in $2 \%$ osmium tetroxide in $0.1 \mathrm{M}$ PBS for $1 \mathrm{~h}$ at $4^{\circ}$ $\mathrm{C}$, dehydrated and included in EPON. Ultra-thin sections were stained in a $1 \%$ uranyl acetate and lead citrate solution, and visualized with a Tecnai SPIRIT Transmission Electron Microscope (FEI Company, Eindhoven, 
The Netherlands) working at an acceleration voltage of $120 \mathrm{KV}$. Electron micrographs were taken from similar regions in the slices. Images were acquired with a Megaview III camera and digitized with the iTEM software (Soft Imaging System). Myelin measurements were obtained with the same program and the g-ratio was determined (axon circumference to myelin circumference). More than 50 randomly selected axons were analyzed per slice, in 3-4 slices per treatment.

\section{Intracellular protein phosphorylation assays by xMAP technology}

The phosphorylation state of different intracellular proteins was evaluated in xMAP assays (Luminex). Mouse cerebellar organotypic cultures were collected in lysis buffer and tested with different antibodies against: total Akt/PKB, total p38/SAPK, total STAT3, total extracellular signal-regulated kinase (ERK)/MAPK, total P70 S6Kinase, total JNK/SAPK1, total activating transcription factor-2 (ATF-2), phosphorylated Akt/PKB (Ser473), phosphorylated Akt/PKB (Thr 308), phosphorylated p38/SAPK (Thr180/Tyr182), phosphorylated STAT3 (Tyr705/Ser727), phosphorylated ERK/MAPK (Thr185/ Tyr187), phosphorylated P70 S6Kinase (Thr412), phosphorylated JNK/SAPK1 (Thr183/Tyr185) and phosphorylated ATF-2 (Thr69/71). Control beads were used to verify calibration and optical integrity of the system. Moreover, a positive and negative antibody control was used in each experiment.

\section{Real-time PCR}

RNA was extracted from the mouse cerebellar organotypic slices after different treatment times using the RNeasy kit, according to the manufacturer's instructions (Qiagen). Using the RNA $(1 \mu \mathrm{g})$ as a template, cDNAs were synthesized (Applied Biosystems) and the following genes were assayed by real-time PCR (Applied Biosystems): Brain derived neurotrophic factor (BDNF), Neurotrophin 3 (NT3), leukemia inhibitory factor (LIF), hairy and enhancer of split 5 (Hes5), CNTF, fibroblast growth factor 2 (FGF-2), mammalian target of rapamycin (mTOR), and nerve growth factor (NGF). To quantify gene expression the comparative CT method was used where the $\triangle \mathrm{CT}$ value was obtained by subtracting the $\mathrm{CT}$ value of an endogenous gene (GAPDH for mouse samples, 18S for human samples) from the CT value of the gene of interest, in each case using a mean value of three reactions.

\section{Data analysis}

The data is expressed as the mean \pm SEM and statistical analyses were performed with SPSS 20.0 software. The data were tested using the paired sample Student $t$-test or with an one-way analysis of variance (ANOVA) and the Bonferroni's $t$-test for multiple comparisons. The relevant statistical results are indicated in the figure legends.

\section{Results \\ Methylthioadenosine promotes remyelination in organotypic cerebellar cultures}

Neuroinflammation is a common process in several neurological diseases, including MS where it leads to demyelination and axonal damage. To assess how methylthioadenosine might affect demyelination/remyelination under inflammatory conditions, we used an in vitro model of neuroinflammation, which was provoked in mouse cerebellar organotypic cultures by stimulation with LPS [16]. We found that the LPS challenge induced microglial/astrocytes activation through the release of the pro-inflammatory cytokines TNF- $\alpha$ and IL-1ß, and the production of iNOS, consequently provoking demyelination and axonal damage (axonal swelling and end bulbs: Fig. 1A, panels b, e, h and j, and B). However, when cultures were pre-treated with methylthioadenosine (48 or $192 \mu \mathrm{M})$, there was significantly less demyelination and no axonal abnormalities were observed (Fig. 1A, panels c, $f, i, j)$. We also observed a significant decrease in IL-1 $\beta$, TNF- $\alpha$ and iNOS expression in methylthioadenosine pretreated cultures (Fig. 1B), as described previously [16], as well as the persistence of CNPase detected in Western blots (Fig. 1B). These results indicate that methylthioadenosine dampened the effects of neuroinflammation, which contributed to the preservation of myelin. However, because the immunomodulatory effects of methylthioadenosine [13], we cannot discriminate in this model whether the preservation of myelin was due to a reduction in inflammatory damage either on microglia or astrocytes activation, or to a direct effect on OLs.

In order to assess the direct effects of methylthioadenosine on remyelination, we used organotypic cerebellar cultures that were challenged with lysolecithin [17]. Following a $7 d$ recovery post-isolation, the slices were treated with lysolecithin $(0.5 \mathrm{mg} / \mathrm{ml})$ for $14 \mathrm{~h}$ to induce demyelination with minimal axonal injury and neuronal damage [17]. Quantification of the g-ratio demonstrated widespread demyelination in the cultures (Fig. 2) and when these demyelinated cultures were treated with methylthioadenosine $(192 \mu \mathrm{M})$, significant remyelination was observed relative to the placebo (saline) treated cultures at all the time points tested $(9,13,17$ and 21 days in vitro). Changes in the g-ratio were observed for nearly all axons, although they were most pronounced for smaller-caliber axons (Fig. 2, panel c). 

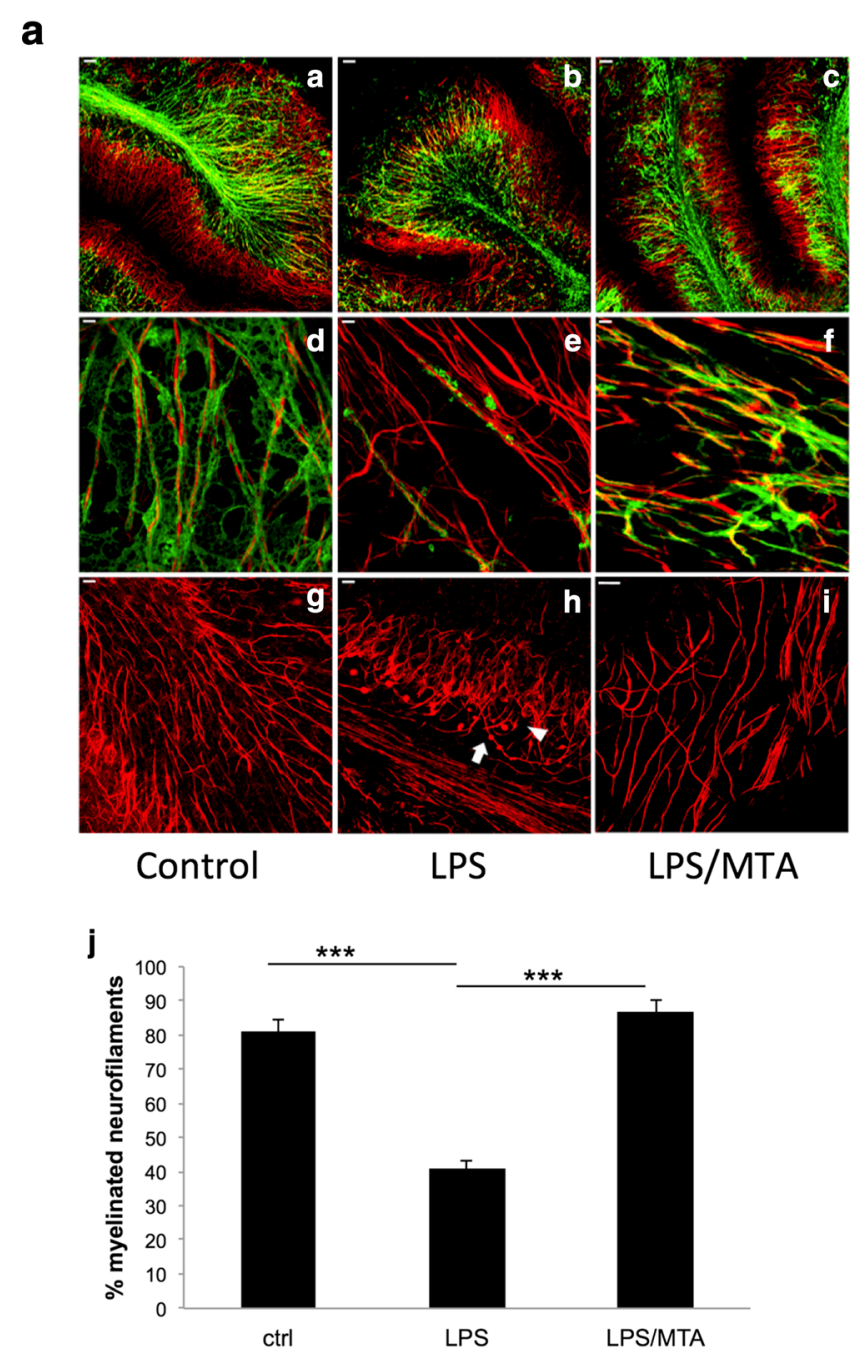

b
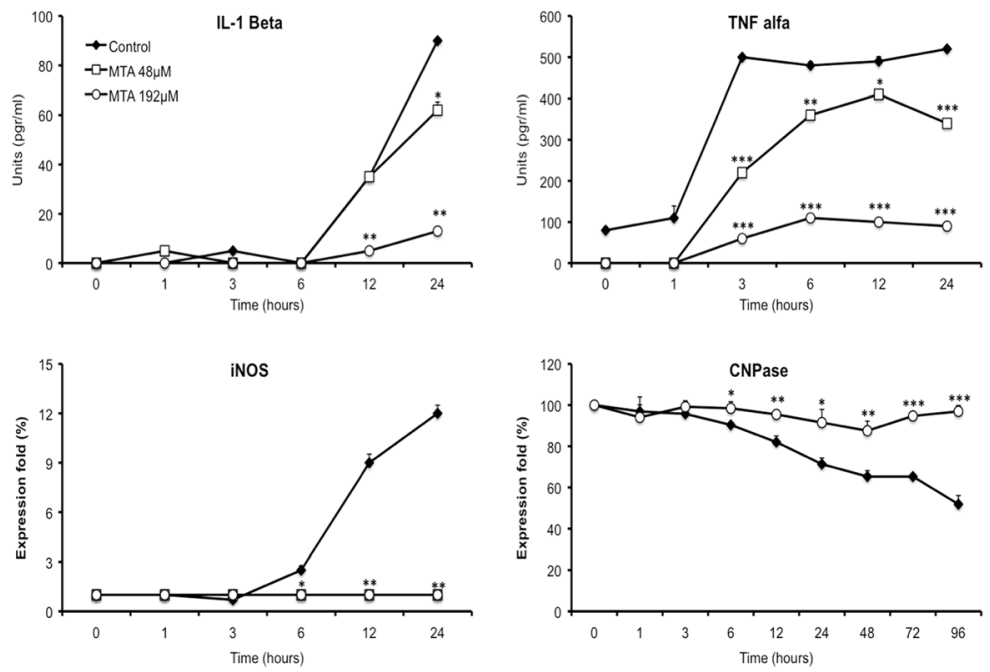

Fig. 1 (See legend on next page.) 
(See figure on previous page.)

Fig. 1 Methylthioadenosine (MTA) dampens neuroinflammation, and prevents demyelination and axonal loss in mouse cerebellar cultures. A Organotypic cultures were treated with MTA $(192 \mu \mathrm{M})$ or the placebo for $24 \mathrm{~h}$ at seven DIV and thereafter, they were stimulated with LPS (15 $\mu \mathrm{g} /$ $\mathrm{ml}$ ) for $24 \mathrm{~h}$. Immunofluorescent staining for NFL (red) and MBP (green) was analyzed in the slices, and in time-matched untreated control slices (arrow, axonal swelling; arrowhead, end bulb). Panels $a, b$ and $c$ scale bars: $50 \mu \mathrm{m}$. Panels $d-i$ scale bar: $5 \mu \mathrm{m}$. Panel $j$ shows the percentage of myelinated neurofilaments. B Cerebellar organotypic cultures at seven DIV were pre-treated with MTA (48 $\mu$ M and $192 \mu$ M) or the placebo and stimulated with LPS $(15 \mathrm{\mu g} / \mathrm{ml})$ at different time points $(0,1,3,6,12,24,48,72$ and $96 \mathrm{~h})$. IL-1 $\beta$ and TNF-a production was measured by ELISA and the cytokine release into the medium is expressed in $\mathrm{pg} / \mathrm{ml}$. The expression of iNOS and CNPase was measured in Western blots. Results are the mean of three independent experiments. ${ }^{*} P<0.05,{ }^{* *} P<0.01$ and ${ }^{* * *} P<0.001$

\section{Methylthioadenosine increases the number of mature myelinating oligodendrocytes in cerebellar organotypic cultures}

Mouse cerebellar organotypic cultures exposed to lysolecithin demyelination contained more mature OLs (Olig2 +
MBP + cells) when they were exposed to methylthioadenosine 1 day after demyelination was induced and when treated with methylthioadenosine in a preventive manner (overnight prior to lysolecithin challenge: Fig. 3a-b). These results indicate that methylthioadenosine promoted OLs

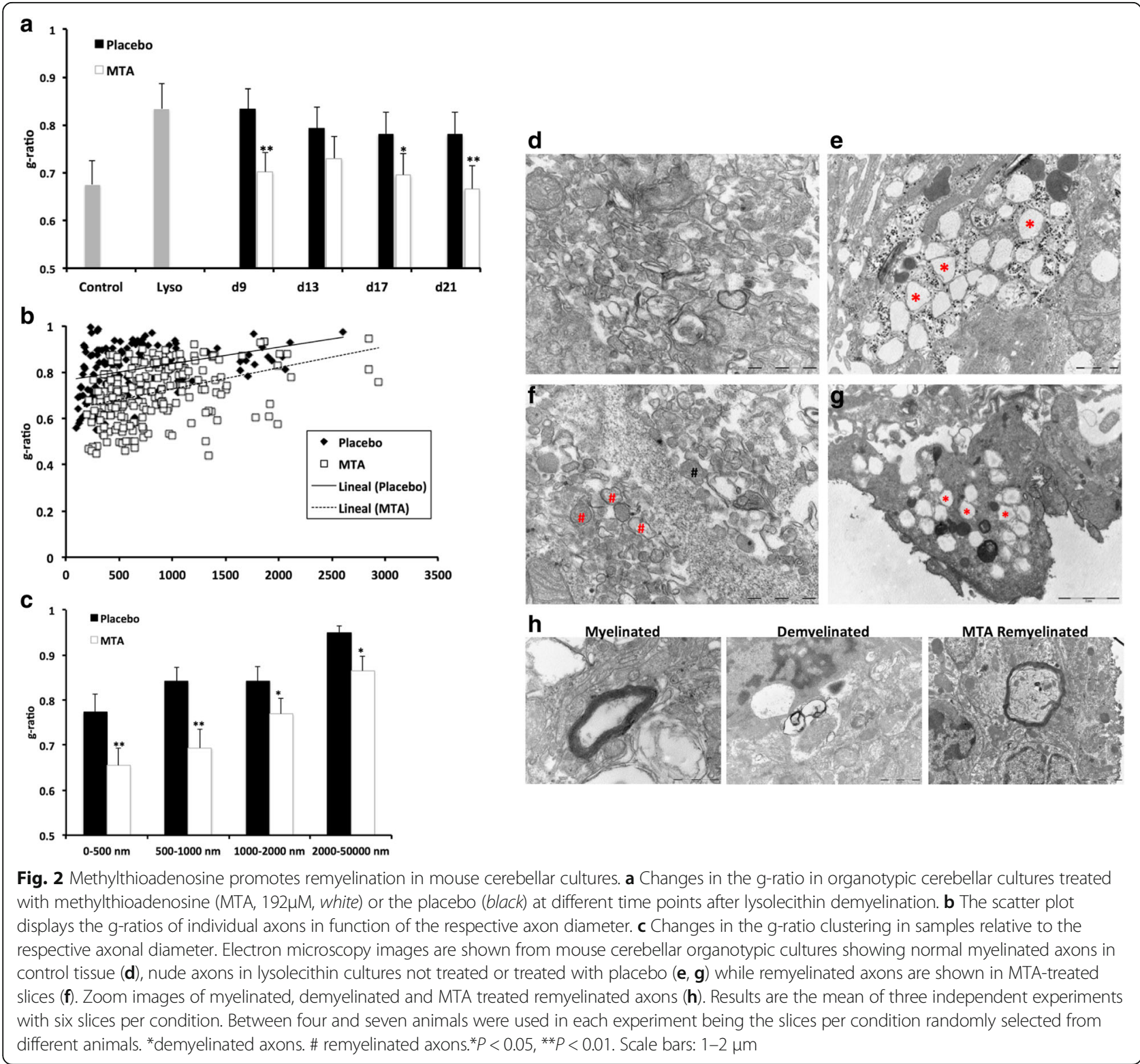



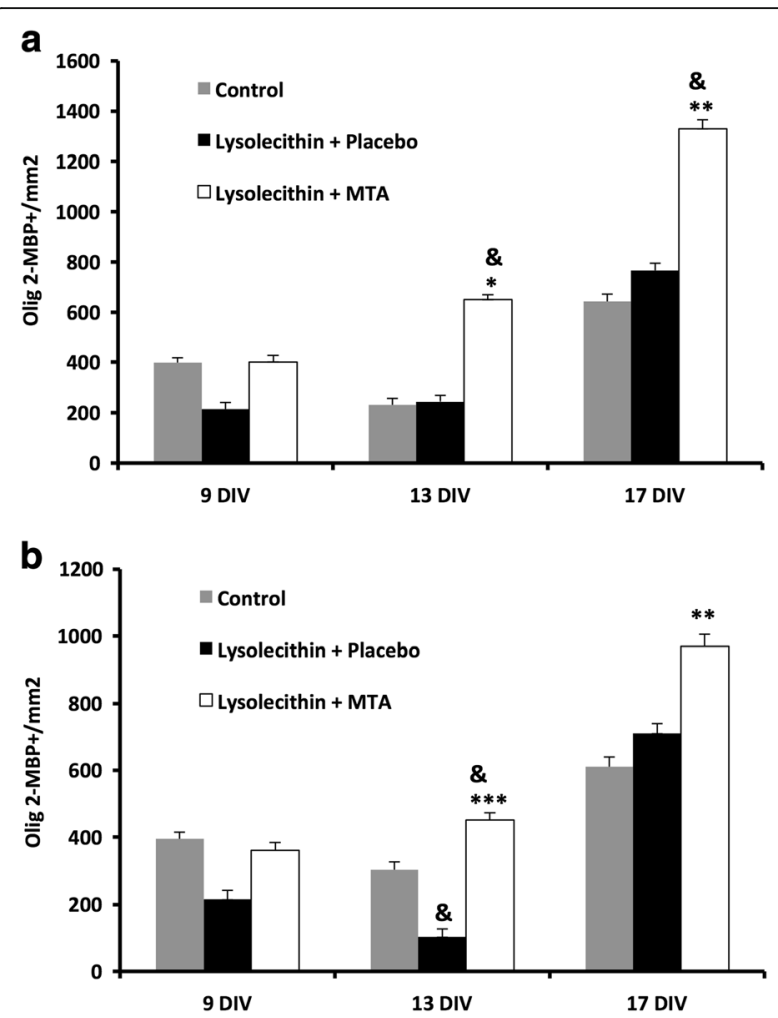

Fig. 3 Methylthioadenosine increases the number of mature myelinating OLs in mouse cerebellar cultures. a Effect of methylthioadenosine (MTA, 192 $\mu \mathrm{M})$ treatment before lysolecithin induced demyelination. Olig2/MBP staining was used as a marker of mature OLs: Grey, control tissue (no lysolecithin); White, lysolecithin challenged tissue treated with MTA at different time points (9, 13 and 17 DIV); Black, lysolecithin challenged tissue treated with the placebo. b Effect of MTA (192 $\mu \mathrm{M})$ on mature myelinating OLs after lysolecithin induced demyelination: Grey, control tissue without lysolecithin; White, lysolecithin tissue treated with MTA; Black, lysolecithin tissue treated with placebo at different time points $(9,13$ and 17 DIV). Results are the mean of three independent experiments with six slices per condition in each experiment. ${ }^{*} p \leq 0.05 ;{ }^{* *} p \leq 0.01$ and ${ }^{* *} p \leq 0.001$ with respect to the placebo; $\& p \leq 0.01$ relative to the controls

survival and differentiation, therefore preventing demyelination and stimulating remyelination.

\section{Methylthioadenosine induces remyelination and} functional recovery in vivo in the cuprizone model We used the cuprizone model of demyelination in mice to assess the effect of methylthioadenosine on remyelination in vivo. We induced demyelination in C57BL6 mice through 4, 5 and 6 weeks of cuprizone intoxication [18]. We followed the remyelination process for 4 weeks after cuprizone removal, administering methylthioadenosine either as a preventive therapy from the same day the mice began to receive cuprizone or as a curative therapy from the 5th week after cuprizone administration had commenced. As expected 4, 5 or 6 weeks of cuprizone treatment significantly diminished the area of
MBP staining in the corpus callosum (Fig. 4a). MBP staining was significantly more extensive at weeks 5 and 6 in animals pretreated with methylthioadenosine (preventive therapy group) than in those that received the vehicle alone (Fig. 4a). In addition, there was stronger MBP staining in animals that received methylthioadenosine (curative therapy group) 2 weeks after cuprizone removal compared to the control animals and those that received the vehicle alone (Fig. 4b). However, there were no differences in MBP staining by weeks 8 and 9 due to the spontaneous remyelination in this model. To assess functional repair, mice were subjected to the rotarod test twice a week from week 5 (upon cessation of the cuprizone supplement) to week 8 . We found significative differences in the motor coordination and balance by week 7 to 8 between methylthioadenosine and vehicle treated animals (Fig. 4c), which corresponds to the time of MBP analysis in the corpus callosum of these animals. We also saw differences at weeks 6 and 7 tested between methylthioadenosine treated animals and cuprizone animals (Fig. 4c).

\section{Methylthioadenosine promotes STAT-3 phosphorylation and CNTF release in cerebellar cultures}

In order to gain insight as to how methylthioadenosine might promote remyelination, we assessed the phosphorylation of key signaling pathways in the mouse cerebellar organotypic model of chemical demyelination with lysolecithin. In this model, the JNK/SAPK pathway normally associated with activation of apoptotic signaling in OLs is activated by the lysolecithin challenge [17]. However, methylthioadenosine dampened the phosphorylation of JNK/SAPK at 13 and 17 DIV, while enhancing the phosphorylation of STAT3 and ERK1/2 compared to that in the slices that received the placebo (Fig. $5 \mathrm{a}-\mathrm{c}$ ). In addition, phosphorylation of the Activating Transcription Factor 2 (ATF2), a transcription factor involved in protection against oxidative stress [19], was also significantly enhanced in cultures exposed to methylthioadenosine after demyelination (Fig. 5d). We did not observe phosphorylation of other relevant kinases such as AKT/ PKB, p38/SAPK or the p70S6 Kinase (data not shown).

A physiological response of the CNS to damage is the release of trophic factors that promote cell survival and restore the CNS microenvironment [20]. For this reason, we performed real-time PCR to measure the expression of genes encoding different trophic factors that could protect OPCs or OLs against damage when the mouse cerebellar slices were challenged with lysolecithin. Of the trophic factors associated with myelin maintenance tested (CNTF, LIF, BDNF, NT3, NGF and FGF-2), only CNTF expression had increased significantly in methylthioadenosine treated slices by day 17 DIV. We also quantified Hes 5 expression, a transcription factor associated with the Notch/Jagged pathway that is implicated in the inhibition of remyelination, and 


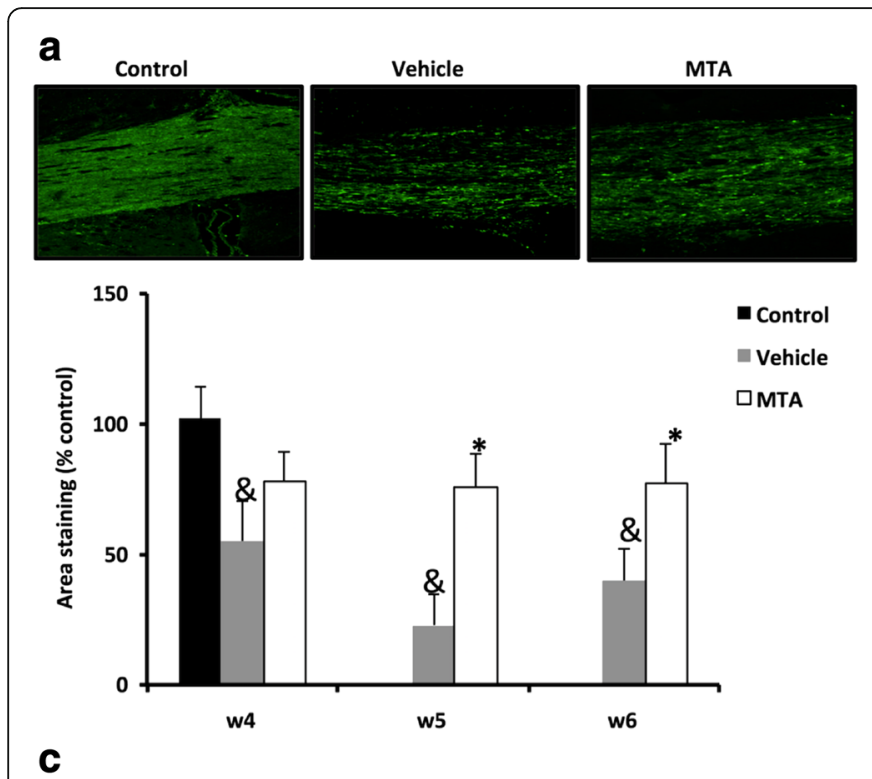

b
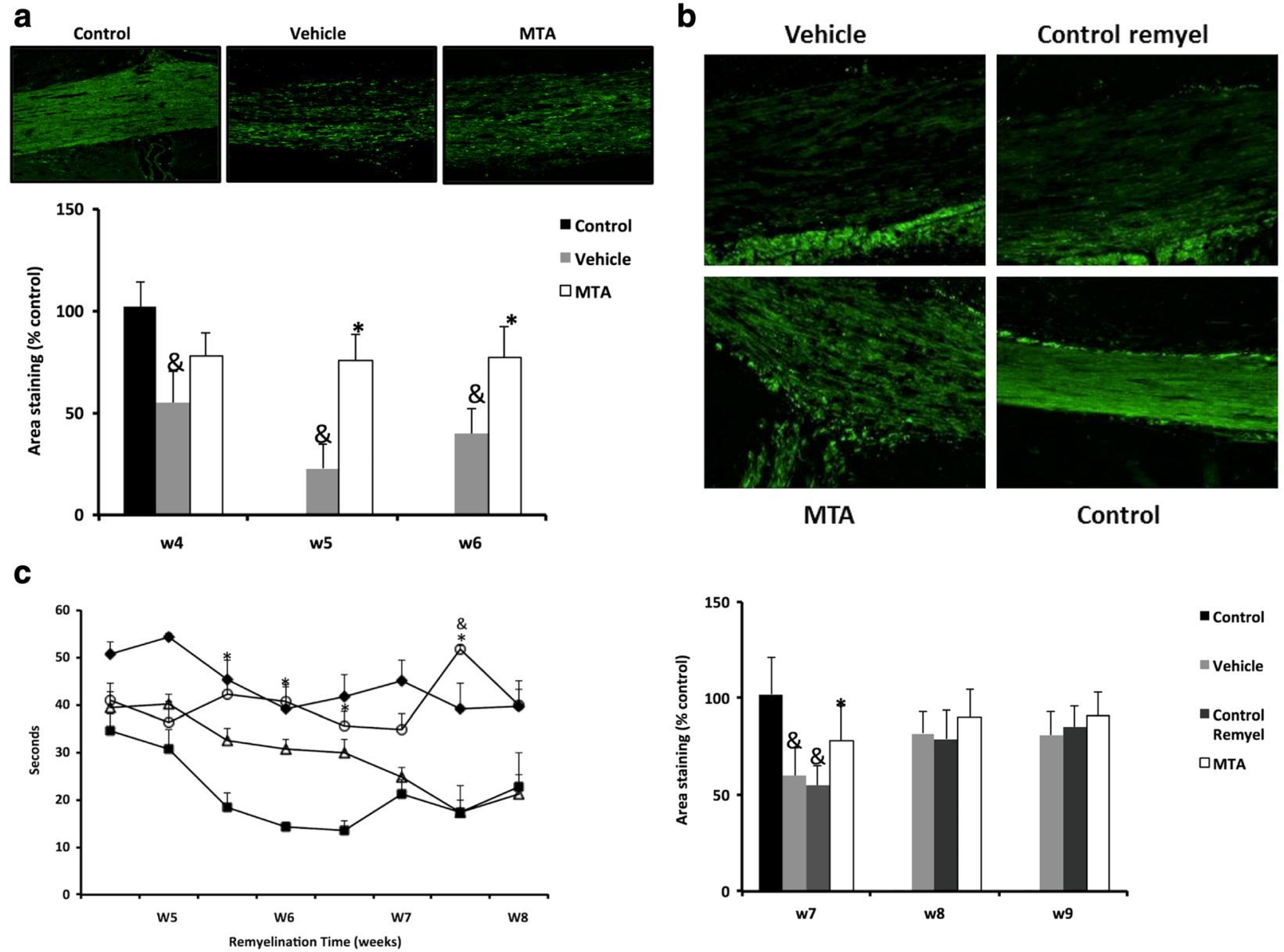

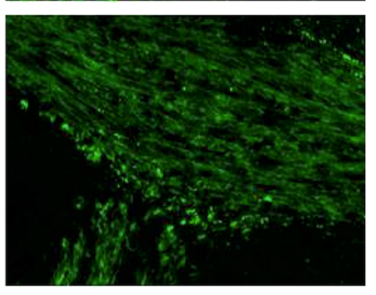

MTA

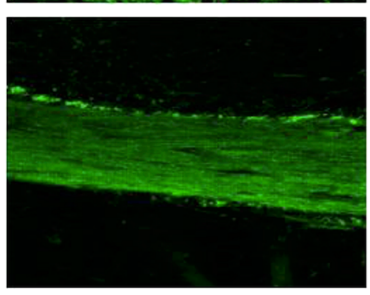

Control

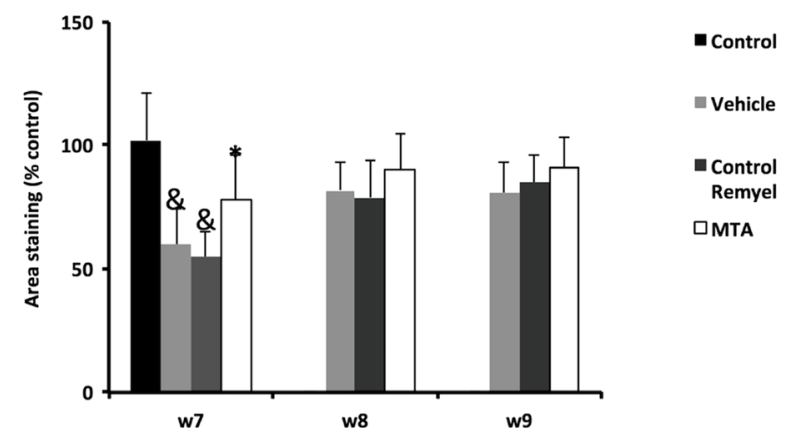

Fig. 4 Methylthioadenosine promotes remyelination in the mouse cuprizone model. a Preventive trial. Top: representative images of MBP immunostaining in the corpus callosum of mice fed with a normal diet (control) or with a cuprizone supplement for 4,5 or 6 weeks and injected intraperitoneally daily with methylthioadenosine (MTA, $60 \mathrm{mg} / \mathrm{kg} /$ day) or the vehicle alone (saline) for 6 weeks (treatments started the same day as cuprizone supplementation). Bottom: quantification of the area of MBP staining shown as the mean \pm standard error of the mean ( $n=6$ per group). b Curative trial. Top: representative images of MBP immunostaining in the corpus callosum of mice fed with normal diet (control) or with a cuprizone supplement for 5 weeks, after which the animals received a normal diet and a daily intraperitoneal injection of the vehicle alone, MTA $(60 \mathrm{mg} / \mathrm{kg} /$ day) or a placebo for 7,8 or 9 weeks. Each treatment started after the cessation of cuprizone supplementation at week 5 . Bottom: quantification of the area stained for MBP. The values shown are the means \pm standard error of the mean ( $n=6$ per group): $\& p \leq 0.05$ respect to control; ${ }^{*} p \leq 0.05$ respect to vehicle. c Clinical score in cuprizone model. Motor coordination and balance were evaluated twice a week from week 5 to week 9 using the rotarod test and expressed as seconds mice stay in the rotarod. Black diamonds, control with no cuprizone; black squares, cuprizone-fed mice; white triangles, cuprizone-fed vehicle treated mice; and white circles, cuprizone-fed and MTA treated mice. The graph represents the average time spent by mice on the rotarod at $18 \mathrm{rpm}$ constant speed. ${ }^{*} p \leq 0.05$ respect to cuprizone fed animals. \& $p \leq 0.05$ respect to vehicle cuprizone fed animals

mTOR, a serine-protein kinase that regulates several pathways involved in cell growth, and survival. We found that neither Hes5 nor mTOR expression changed significantly in cultures exposed to methylthioadenosine (Additional file 2: Figure S2).

To confirm the increase in STAT- 3 phosphorylation in the organotypic cultures and study the sequence of events implicated in remyelination induced by methylthioadenosine, we exposed cerebellar cultures challenged with lysolecithin to the inhibitor of STAT-3 phosphorylation, LLL12. This inhibitor partially inhibited STAT-3 phosphorylation in these cultures, an effect that was reversed by methylthioadenosine (Fig. 6a). When we assessed the expression of the CNTF gene when STAT3 phosphorylation was blocked, there was a significant decrease in CNTF expression in cultures that received LLL12 but not in those that were also administered methylthioadenosine (Fig. 6b). In summary, the effects of methylthioadenosine in cerebellar cultures were associated with the phosphorylation of STAT3 and other kinases, and with the expression of CNTF. 
a

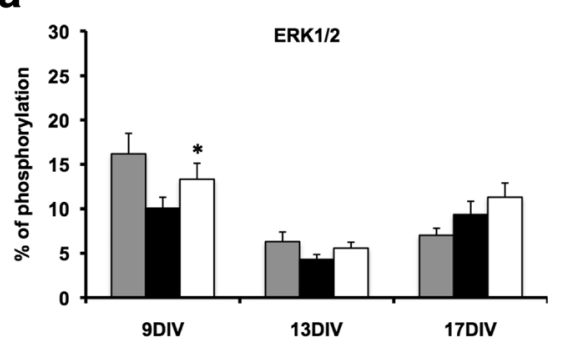

C

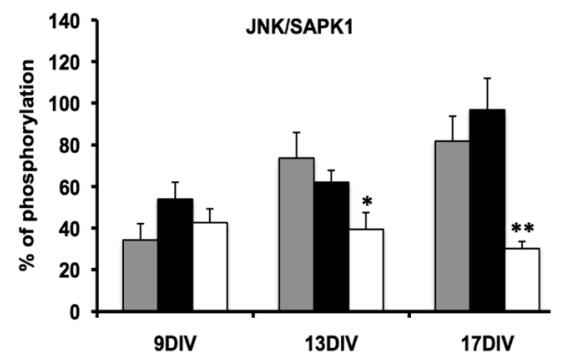

b

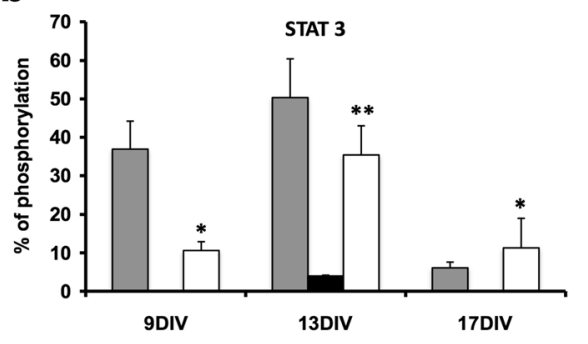

d

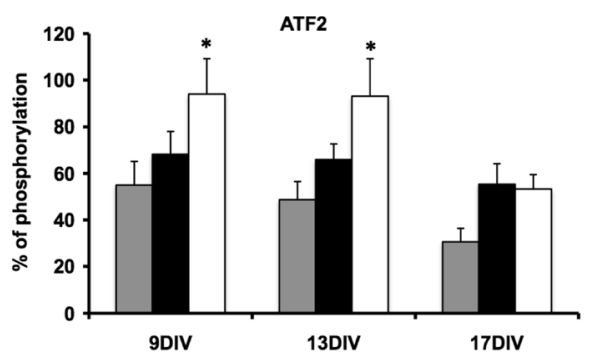

Fig. 5 Activation of signaling pathways involved in remyelination. Intracellular ERK1/2 (a), STAT3 (b), JNK/SAPK1 (c) and ATF2 (d) phosphoproteins measured in XMAP assays (Luminex) of mouse cerebellum cultures exposed to lysolecithin $(0.5 \mathrm{mg} / \mathrm{ml}$ ) and treated with methylthioadenosine (MTA, $192 \mu \mathrm{M})$ or the placebo at 9, 13 and 17 DIV. Samples for the phosphorylation assay were obtained $30 \mathrm{~min}$ after the last administration of MTA or the placebo. Results are the mean of three independent experiments with 10 slices per condition in each experiment. Gray, Control samples without lysolecithin; Black, Lysolecithin samples treated with placebo; White, Lysolecithin samples treated with MTA; * $p<0.05$ and ${ }^{* *} p<0.01$ with respect to the samples that received the placebo

\section{Role of astrocytes in Methylthioadenosine mediated remyelination}

In order to identify the source of CNTF promoted by MTA, we performed immunohistochemistry on the mouse cerebellum slices. We observed a co-localization of GFAP with CNTF, suggesting that astrocytes are the main producers of CNTF in these cultures (Fig. 6c). We also used mouse primary cortical astrocyte cultures stimulated with LPS to validate the role of methylthioadenosine in promoting the expression of CNTF by astrocytes. Astrocytes exposed to methylthioadenosine for $24 \mathrm{~h}$ produced significantly more CNTF RNA (Fig. 7a) and protein (Fig. 7b). Moreover, STAT-3 phosphorylation in mouse astrocyte cultures challenged with LPS increased significantly $30 \mathrm{~min}$ and $1 \mathrm{~h}$ after methylthioadenosine treatment (Fig. 7c).

\section{Discussion}

In this study, we evaluated the ability of methylthioadenosine, a natural metabolite, to promote remyelination and protect against demyelination in models of inflammatory and chemical demyelination. Intermediate metabolism plays a critical role in maintaining cell homeostasis and in the response to stress. For example, an intermediate metabolite of the Kreb's cycle, fumarate acid, displays antioxidant properties by increasing the expression of Nrf2, a transcription factor that induces the expression of antioxidant enzymes [21]. Kynurenines or the methylthioadenosine precursor S-Adenosyl-Methionine are other examples, both able to modulate cell proliferation and survival in different pathological situations [22-24]. In this study we provide in vitro and in vivo evidence that methylthioadenosine promotes remyelination and protects against demyelination in two different model of demyelination. First, methylthioadenosine promotes remyelination and prevents demyelination in mouse cerebellar cultures exposed to LPS (a model of inflammatory demyelination) and promotes remyelination when exposed to lysolecithin (a model of chemical demyelination), increasing the number of mature myelinating OLs. In the in vivo model of demyelination with cuprizone, methylthioadenosine was able to prevent demyelination and also enhanced remyelination 2 weeks after starting the treatment, as reflected by a clinical improvement in locomotion in this period of time. In this study we did not use the experimental autoimmune encephalitis model because the immunomodulatory activity of methylthioadenosine would prevent the emergence of its effect in demyelination/remyelination.

In mediating these effects on myelination, methylthioadenosine appears to employ a complex and pleiotropic mechanism of action, even though it is a well-documented antioxidant $[9,13,25]$. In this study, we show that methylthioadenosine dampens the expression of iNOS and it enhances the phosphorylation of ATF2, a factor that protects against oxidative stress [19]. Indeed, this finding agrees with previous studies showing that methylthioadenosine can counteract oxidative stress in other cell types and settings [9, 

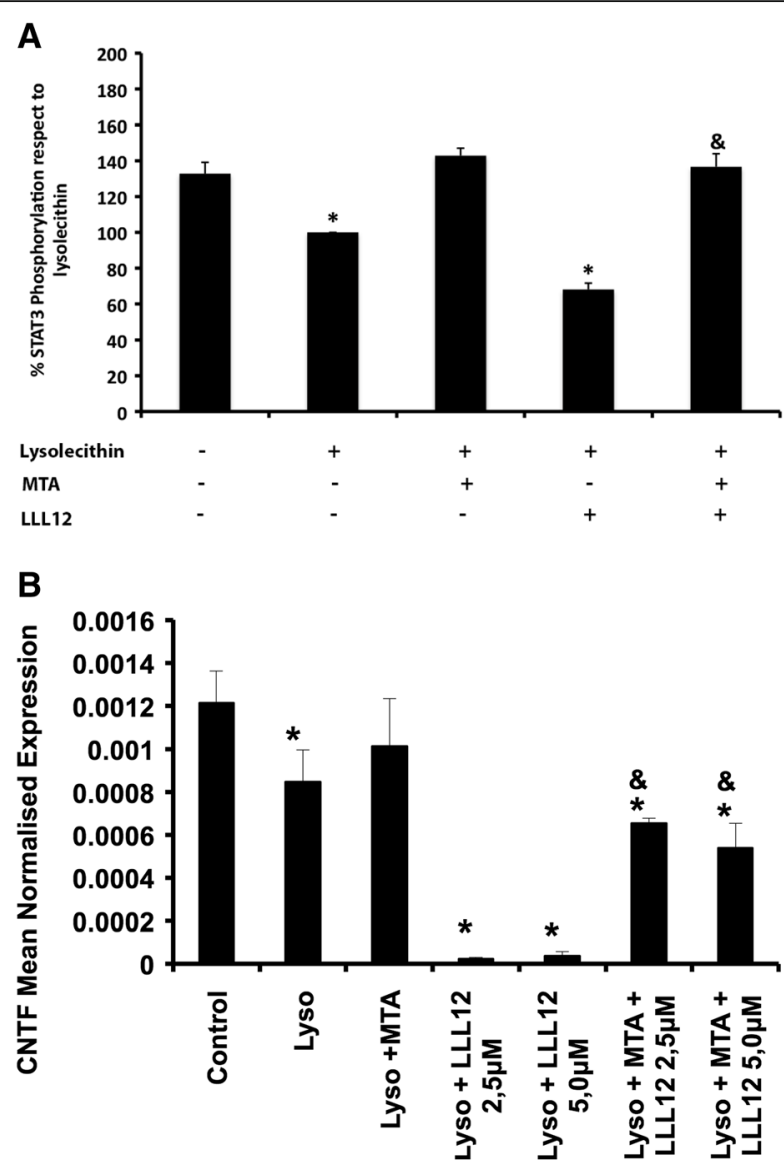

C

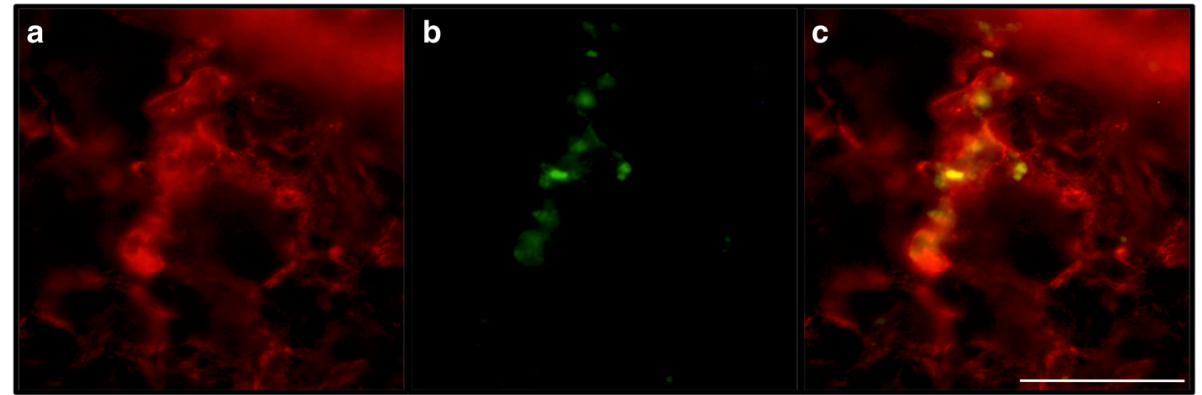

Fig. 6 Modulation of signaling pathways associated with remyelination and cell survival. A Proportion of intracellular phospho-STAT-3 (Tyr705/Ser727) measured in XMAP assays of cerebellar cultures exposed to lysolecithin $(0.5 \mathrm{mg} / \mathrm{ml})$ and treated with MTA (192 $\mu$ M) or the STAT3 inhibitor LLL12 (5 $\mu \mathrm{M})$ for 4 days. The cells were processed $30 \mathrm{~min}$ after MTA or LLL12 exposure on the last day of experiment: ${ }^{*} P<0.05$, statistical differences respect to control without lysolecithin; $P<0.05$, statistical differences respect to Lysolecithin + LLL12 (no MTA). B CNTF gene expression in cerebellar organotypic cultures exposed to lysolecithin $(0.5 \mathrm{mg} / \mathrm{ml})$ and treated with MTA $(192 \mu \mathrm{M})$ or LLL12 $(2.5 \mu \mathrm{M}$ or $5 \mu \mathrm{M})$ ) ${ }^{*} P<0.05$, statistical differences relative to the control without lysolecithin; \& $P<0.05$, statistical differences relative to Lysolecithin + LLL12 (no MTA). C Immunohistochemistry for GFAP (red) and

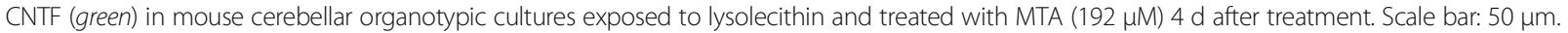
Results are the mean of three independent experiments with 10 slices per condition in each experiment

13, 25]. Here, we also show that methylthioadenosine can down-regulate microglia/astrocytes activation, inhibiting the release of pro-inflammatory cytokines like TNF- $\alpha$ and IL-1 $\beta$. Microglia/astrocytes activation apparently plays a critical role in amplifying brain damage and has been considered an interesting therapeutic strategy in many brain diseases [26].
Methylthioadenosine also promotes STAT-3 phosphorylation in the mouse cerebellum organotypic cultures exposed to lysolecithin, a signaling protein previously associated with neurite outgrowth [27], axonal regeneration and survival [28]. It should be noted that STAT-3 expression and activation in the CNS is very specific 
a

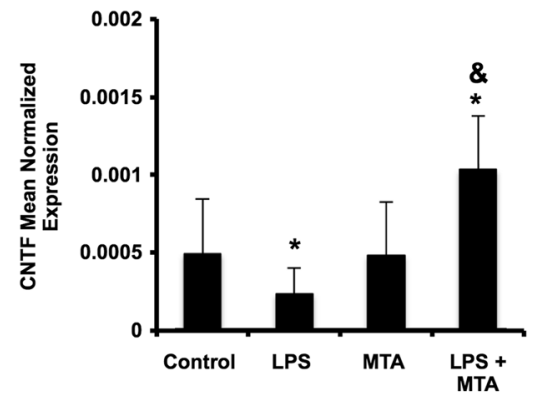

C

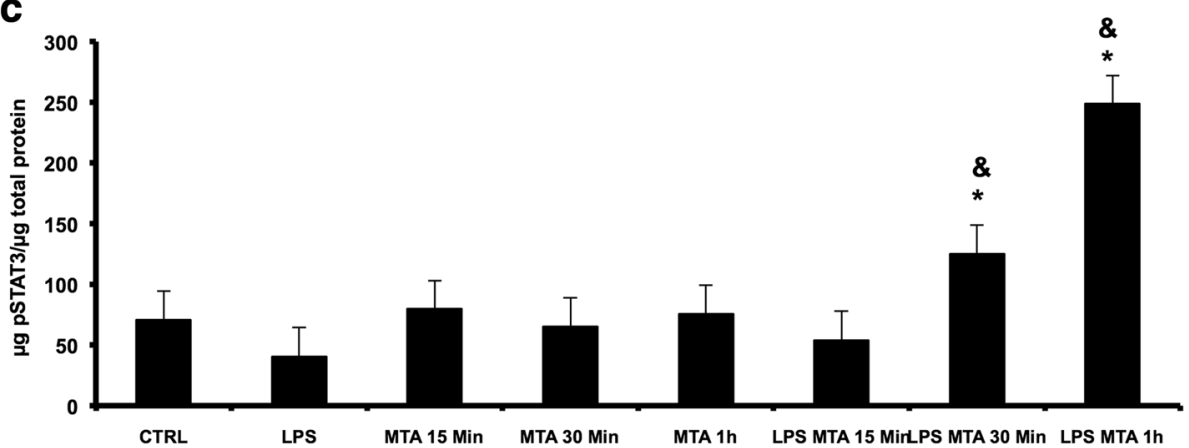

b

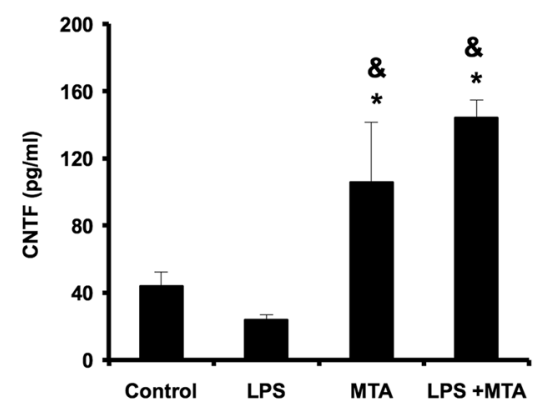

Fig. 7 Role of methylthioadenosine in CNTF production and STAT-3 phosphorylation in astrocytes. a CNTF gene expression in primary cortical mouse astrocytes exposed to LPS $(10 \mu \mathrm{g} / \mathrm{ml})$ overnight and treated for $24 \mathrm{~h}$ with methylthioadenosine (MTA, 192 $\mu \mathrm{M})$ or the placebo on the following day. $\mathbf{b}$ CNTF protein measured by ELISA in supernatants of primary cortical mouse astrocytes exposed to LPS (10 $\mu \mathrm{g} / \mathrm{ml})$ overnight and treated for $24 \mathrm{~h}$ with MTA $(192 \mu \mathrm{M})$ or placebo the following day. c Intracellular phospho-STAT-3 (Tyr705/Ser727) levels measured in xMAP assays carried out on primary

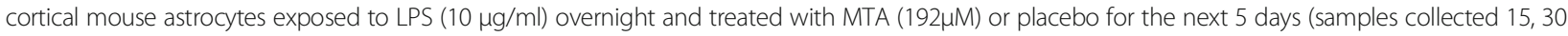
min or $1 \mathrm{~h}$ after the last treatment was given): ${ }^{*} p<0.05$, statistical differences relative to the controls that did not receive LPS; $p<0.05$, statistical differences relative to the cells that received LPS but not MTA. Results are the mean of two independent experiments

given that it can fulfill a number of different roles both in the developing and adult brain, many related to neuroprotection [29]. The enhanced STAT-3 phosphorylation induced by methylthioadenosine in the CNS was associated with the release of CNTF by astrocytes, as demonstrated using a partial and reversible chemical inhibitor of STAT3 phosphorylation in organotypic cultures. However, understanding the mechanisms by which methylthioadenosine promotes STAT-3 phosphorylation and the release of CNTF will require further studies.

CNTF promotes remyelination and it enhances the survival and differentiation of CNS-derived oligodendroglia [30-33]. The identification of pathways that control OPC differentiation and myelination has paved the way for the development of new approaches to achieve remyelination [34]. CNTF is produced by microglia and astrocytes following brain injury $[35,36]$, and it enhances the survival and differentiation of OLs [30-33]. Finally, methylthioadenosine is signalling through adenosine receptors [37], and activation of adenosine receptor in OPCs promote myelination [38]. Therefore, methylthioadenosine might contribute to myelin repair through activation of adenosine pathway as well. Further studies are required to understand the role of each pathway involved in the remyelinating activity we have observed.

\section{Conclusion}

Based on these results, we propose that methylthioadenosine may offer a natural means to obtain cell protection and to achieve repair in circumstances of CNS damage. Methylthioadenosine therapy may recapitulate such responses and also provides benefits by limiting brain damage in neuroinflammation. The results of this study provide the opportunity to test methylthioadenosine as a regenerative therapy for neuroinflammatory diseases like MS. Accordingly, methylthioadenosine could be integrated in a combination therapy for MS taking advantage of the immunomodulatory activity of current drugs along with methylthioadenosine's immunomodulatory and regenerative effects. Indeed, methylthioadenosine has already proven to be safe in previous phase I studies [39], a critical consideration when developing combination therapies for autoimmune diseases. 


\section{Additional files}

Additional file 1: Figure S1. Experimental design cuprizone model. Cuprizone induced demyelination was induced by feeding mice with cuprizone from week 0 to week 6 . MTA was tested in as preventive therapy in order to decrease demyelination (from week 0 to week 6) or as curative therapy in order to promote remyelination (from week 5 until week 9). Placebo animals were also feed with cuprizone and were treated with placebo (Plac); control animals did not received cuprizone. (TIFF 806 kb)

Additional file 2: Figure S2. Expression of trophic factors after in vitro demyelination in cerebellar organotypic cultures. The expression of genes encoding trophic factors such as LIF, Hes5, BDNF, NT3, CNTF, NGF, mTOR and FGF-2 was assessed in mouse cerebellum cultures exposed to lysolecithin (0.5 $\mathrm{mg} / \mathrm{ml})$ and treated with MTA $(192 \mu \mathrm{M})$ or the placebo at 9, 13, 17 and 21 DIV by real-time PCR. The data represent the increase relative to the housekeeping gene GAPDH. Results are the mean of three independent experiments with 10 slices per condition in each experiment. * $p<0.05$. (TIFF $445 \mathrm{~kb}$ )

\section{Abbreviations}

ANOVA: One-way analysis of variance; BDNF: Brain derived neurotrophic factor; BSA: Bovine serum albumin; CNPase: 2',3'-cyclic-nucleotide 3'phosphodiesterase; CNS: Central Nervous System; CNTF: Ciliary neurotrophic factor; DIV: Days in vitro; FGF-2: Fibroblast growth factor 2; GFAP: Glial fibrillary acidic protein; Hes5: Hairy and enhancer of split 5; iNOS: Inducible nitric oxide synthase; ip: Intraperitoneal; LIF: Leukemia inhibitory factor; LPS: Lipopolysaccharide; MBP: Myelin basic protein; MS: Multiple sclerosis; mTOR: Mammalian target of rapamycin; NFkB: Nuclear factor kappa B; NFL: Neurofilament; NGF: Nerve growth factor; NT3: Neurotrophin 3; OLs: Oligodendrocytes; OPCs: Oligodendrocyte precursor cells; PRMT5: Protein arginine methyltransferase 5

\section{Acknowledgement}

We would like to thank Mark Sefton for the English revision.

\section{Funding}

This work was supported by grants PI12/01823 and RD07/60/001 to PV from the Instituto de Salud Carlos III, Spanish Ministry of Economy and Competitivity (Spain) and CERCA Programme / Generalitat de Catalunya. Funding agency has no role in the design, analysis and writing of the article.

\section{Availability of data and material}

Raw data is available from authors upon request

\section{Author's contributions}

PV and BM designed the research plan, analyzed the results and wrote the manuscript. BM, AdP, GV, RV, OE, NE and BF performed the organotypic culture assays, and the mechanism of action studies. AM and JA performed the astrocyte assays. All authors read and approved the final manuscript.

\section{Competing interests}

PV and BM hold patent rights on the use of methylthioadenosine for the treatment of MS and other neurological diseases. PV has received consultancy honoraria from Digna Biotech (Pamplona, Spain).

\section{Consent for publication}

Not applicable

\section{Ethics approval}

The animal protocols used were approved by the Ethical Committee on Animal Research at the University of Barcelona.

\section{Publisher's Note}

Springer Nature remains neutral with regard to jurisdictional claims in published maps and institutional affiliations.

\section{Author details}

'Center of Neuroimmunology, Institut d'Investigacions Biomediques August Pi Sunyer (IDIBAPS), Barcelona, Spain. ${ }^{2}$ Department of Basic Sciences.
Facultad de Medicina i Ciències de la Salut, Universitat International de Catalunya (UIC), Sant Cugat del Vallés, Spain. ${ }^{3}$ Neurogenomiks, University of Basque Country, Leioa, Spain. ${ }^{4}$ University of Barcelona, Barcelona, Spain. ${ }^{5}$ University of California, San Francisco, USA. ${ }^{6}$ Centre Cellex 3A, Casanova 145, 08036 Barcelona, Spain.

Received: 14 December 2016 Accepted: 7 March 2017

Published online: 14 April 2017

\section{References}

1. Ransohoff RM, Hafler DA, Lucchinetti CF. Multiple sclerosis-a quiet revolution. Nat Rev Neurol. 2015;11(3):134-42.

2. Hauser SL, Chan JR, Oksenberg JR. Multiple sclerosis: prospects and promise. Ann Neurol. 2013;74(3):317-27.

3. Lubetzki C, Stankoff B. Demyelination in multiple sclerosis. Handb Clin Neurol. 2014;122:89-99.

4. Nave KA, Werner HB. Myelination of the nervous system: mechanisms and functions. Annu Rev Cell Dev Biol. 2014;30:503-33.

5. Luessi F, Kuhlmann T, Zipp F. Remyelinating strategies in multiple sclerosis. Expert Rev Neurother. 2014;14(11):1315-34.

6. Franklin RJ, ffrench-Constant C, Edgar JM, Smith KJ. Neuroprotection and repair in multiple sclerosis. Nat Rev Neurol. 2012;8(11):624-34.

7. Charles P, Reynolds R, Seilhean D, Rougon G, Aigrot MS, Niezgoda A, et al. Re-expression of PSA-NCAM by demyelinated axons: an inhibitor of remyelination in multiple sclerosis? Brain. 2002;125(Pt 9):1972-9.

8. Williams-Ashman HG, Seidenfeld J, Galletti P. Trends in the biochemical pharmacology of 5'-deoxy-5'-methylthioadenosine. Biochem Pharmacol. 1982;31(3):277-88.

9. Avila MA, Garcia-Trevijano ER, Lu SC, Corrales FJ, Mato JM. Methylthioadenosine. Int J Biochem Cell Biol. 2004;36(11):2125-30.

10. Mavrakis KJ, McDonald 3rd ER, Schlabach MR, Billy E, Hoffman GR, deWeck A, et al. Disordered methionine metabolism in MTAP/CDKN2A-deleted cancers leads to dependence on PRMT5. Science. 2016;351(6278):1208-13.

11. Kryukov GV, Wilson FH, Ruth JR, Paulk J, Tsherniak A, Marlow SE, et al. MTAP deletion confers enhanced dependency on the PRMT5 arginine methyltransferase in cancer cells. Science. 2016;351(6278):1214-8.

12. Andreu-Perez P, Esteve-Puig R, de Torre-Minguela C, Lopez-Fauqued M, Bech-Serra JJ, Tenbaum S, et al. Protein arginine methyltransferase 5 regulates ERK1/2 signal transduction amplitude and cell fate through CRAF. Sci Signal. 2011;4(190):ra58.

13. Moreno B, Hevia H, Santamaria M, Sepulcre J, Munoz J, Garcia-Trevijano ER, et al. Methylthioadenosine reverses brain autoimmune disease. Ann Neurol. 2006;60:323-34.

14. Moreno B, Fernandez-Diez B, Di Penta A, Villoslada P. Preclinical studies of methylthioadenosine for the treatment of multiple sclerosis. Mult Scler. 2010;16(9):1102-8

15. Moreno B, Lopez I, Fernandez-Diez B, Gottlieb M, Matute C, Sanchez-Gomez $M V$, et al. Differential neuroprotective effects of 5'-deoxy-5'methylthioadenosine. PLoS One. 2014;9(3):e90671.

16. Di Penta A, Moreno B, Reix S, Fernandez-Diez B, Villanueva M, Errea O, et al. Oxidative stress and proinflammatory cytokines contribute to demyelination and axonal damage in a cerebellar culture model of neuroinflammation. PloSONE. 2013;8(2):e54722.

17. Birgbauer $E$, Rao TS, Webb M. Lysolecithin induces demyelination in vitro in a cerebellar slice culture system. J Neurosci Res. 2004;78(2):157-66.

18. Liebetanz D, Merkler D. Effects of commissural de- and remyelination on motor skill behaviour in the cuprizone mouse model of multiple sclerosis. Exp Neurol. 2006;202(1):217-24.

19. Kiryu-Seo S, Ohno N, Kidd GJ, Komuro H, Trapp BD. Demyelination increases axonal stationary mitochondrial size and the speed of axonal mitochondrial transport. J Neurosci. 2010;30(19):6658-66.

20. Villoslada P, Genain CP. Role of nerve growth factor and other trophic factors in brain inflammation. Prog Brain Res. 2004;146:403-14.

21. Linker RA, Lee DH, Ryan S, van Dam AM, Conrad R, Bista P, et al. Fumaric acid esters exert neuroprotective effects in neuroinflammation via activation of the Nrf2 antioxidant pathway. Brain. 2011;134(Pt 3):678-92.

22. Lu SC, Ramani K, Ou X, Lin M, Yu V, Ko K, et al. S-adenosylmethionine in the chemoprevention and treatment of hepatocellular carcinoma in a rat model. Hepatology. 2009;50(2):462-71.

23. Luo J, Li YN, Wang F, Zhang WM, Geng X. S-adenosylmethionine inhibits the growth of cancer cells by reversing the hypomethylation status of $c$ - 
myc and $\mathrm{H}$-ras in human gastric cancer and colon cancer. Int J Biol Sci. 2010;6(7):784-95.

24. Sahin M, Sahin E, Gumuslu S, Erdogan A, Gultekin M. Inhibition of angiogenesis by S-adenosylmethionine. Biochem Biophys Res Commun. 2011:408(1):145-8.

25. Simile MM, Banni S, Angioni E, Carta G, De Miglio MR, Muroni MR, et al. 5'Methylthioadenosine administration prevents lipid peroxidation and fibrogenesis induced in rat liver by carbon-tetrachloride intoxication. J Hepatol. 2001;34(3):386-94.

26. Moreno B, Jukes JP, Vergara-lrigaray N, Errea O, Villoslada P, Perry VH, et al. Systemic inflammation induces axon injury during brain inflammation. Ann Neurol. 2011;70(6):932-42.

27. He JC, Gomes I, Nguyen T, Jayaram G, Ram PT, Devi LA, et al. The G alpha(o/i)coupled cannabinoid receptor-mediated neurite outgrowth involves Rap regulation of Src and Stat3. J Biol Chem. 2005;280(39):33426-34.

28. Sun F, Park KK, Belin S, Wang D, Lu T, Chen G, et al. Sustained axon regeneration induced by co-deletion of PTEN and SOCS3. Nature. 2011;480(7377):372-5.

29. Dziennis S, Alkayed NJ. Role of signal transducer and activator of transcription 3 in neuronal survival and regeneration. Rev Neurosci. 2008;19(4-5):341-61.

30. Barres BA, Hart IK, Coles HS, Burne JF, Voyvodic JT, Richardson WD, et al. Cell death and control of cell survival in the oligodendrocyte lineage. Cell. 1992;70(1):31-46.

31. Barres BA, Schmid R, Sendnter M, Raff MC. Multiple extracellular signals are required for long-term oligodendrocyte survival. Development. 1993;118(1):283-95.

32. Louis JC, Magal E, Takayama S, Varon S. CNTF protection of oligodendrocytes against natural and tumor necrosis factor-induced death. Science. 1993;259(5095):689-92.

33. D'Souza SD, Alinauskas KA, Antel JP. Ciliary neurotrophic factor selectively protects human oligodendrocytes from tumor necrosis factor-mediated injury. J Neurosci Res. 1996;43(3):289-98.

34. Chari DM. Remyelination in multiple sclerosis. Int Rev Neurobiol. 2007:79:589-620

35. Moore CS, Abdullah SL, Brown A, Arulpragasam A, Crocker SJ. How factors secreted from astrocytes impact myelin repair. J Neurosci Res. 2011;89(1):13-21.

36. Tanaka T, Murakami K, Bando Y, Yoshida S. Minocycline reduces remyelination by suppressing ciliary neurotrophic factor expression after cuprizone-induced demyelination. J Neurochem. 2013;127(2):259-70.

37. Keyel PA, Romero M, Wu W, Kwak DH, Zhu Q, Liu X, et al. Methylthioadenosine reprograms macrophage activation through adenosine receptor stimulation. PLoS One. 2014;9(8):e104210.

38. Stevens B, Porta S, Haak LL, Gallo V, Fields RD. Adenosine: a neuron-glial transmitter promoting myelination in the CNS in response to action potentials. Neuron. 2002;36(5):855-68.

39. Stramentinoli G, Gennari F, inventors; Adenosine derivatives of antiinflammatory and analgesic activity, and therapeutic compositions which contain them as their active principle. US patent 4,454,122. 19841984

\section{Submit your next manuscript to BioMed Central and we will help you at every step:}

- We accept pre-submission inquiries

- Our selector tool helps you to find the most relevant journal

- We provide round the clock customer support

- Convenient online submission

- Thorough peer review

- Inclusion in PubMed and all major indexing services

- Maximum visibility for your research

Submit your manuscript at www.biomedcentral.com/submit

) Biomed Central 